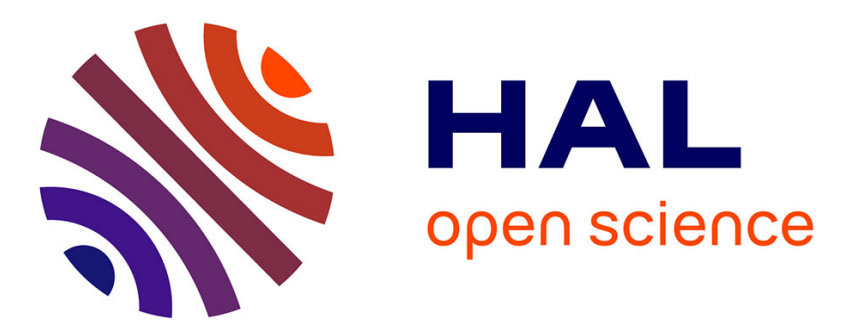

\title{
Propriétés des défauts de surface produits par recuit laser continu sur GaAs
}

\author{
G. Marrakchi, E. Rosencher, M. Gavand, G. Guillot, A. Nouailhat
}

\section{To cite this version:}

G. Marrakchi, E. Rosencher, M. Gavand, G. Guillot, A. Nouailhat. Propriétés des défauts de surface produits par recuit laser continu sur GaAs. Revue de Physique Appliquée, 1987, 22 (11), pp.1451-1458. 10.1051/rphysap:0198700220110145100 . jpa-00245694

\section{HAL Id: jpa-00245694 https://hal.science/jpa-00245694}

Submitted on 1 Jan 1987

HAL is a multi-disciplinary open access archive for the deposit and dissemination of scientific research documents, whether they are published or not. The documents may come from teaching and research institutions in France or abroad, or from public or private research centers.
L'archive ouverte pluridisciplinaire HAL, est destinée au dépôt et à la diffusion de documents scientifiques de niveau recherche, publiés ou non, émanant des établissements d'enseignement et de recherche français ou étrangers, des laboratoires publics ou privés. 


\title{
Propriétés des défauts de surface produits par recuit laser continu sur GaAs
}

\author{
G. Marrakchi, E. Rosencher (*), M. Gavand, G. Guillot et A. Nouailhat
}

Laboratoire de physique de la matière, Institut national des sciences appliquées de Lyon, 20, avenue AlbertEinstein, 69621 Villeurbanne Cedex, France

(*) CNS CNET, chemin du Vieux Chêne, B.P. 98, 38243 Meylan Cedex, France

(Reçu le 16 janvier 1987, révisé le 30 mars 1987, accepté le 26 mai 1987)

\begin{abstract}
Résumé. - Des irradiations par faisceaux laser continu ont été faites sur GaAs dans une gamme de puissances comprises entre 0,5 et 0,8 Pm. Pm étant la puissance de fusion. Les résultats obtenus par DLTS montrent la formation à la surface d'un continuum de pièges notés PL1. Des expériences complémentaires effectuées par ellipsométrie et SIMS permettent de relier les pièges à des défauts dans une faible couche d'oxyde formée durant le traitement. Les expériences de DLTS et DLOS réalisées dans différentes conditions de polarisation 's'interprètent par une relaxation de ces défauts sensibles au champ électrique.
\end{abstract}

\begin{abstract}
C. W. laser annealing on GaAs was performed using incident powers ranging from $0.5 \mathrm{Pm}$ and $0.8 \mathrm{Pm}$. Pm being the melting power. DLTS results show that a trap continuum PL1 is induced in a thin oxyde layer as revealed by ellipsometry and SIMS. These defects have an interesting electrical field effect tentatively explained by a relaxation function of the electrical field.
\end{abstract}

\section{Introduction.}

L'emploi des faisceaux d'électrons ou laser pour recuire les défauts introduits dans les semiconducteurs lors d'une implantation ionique a fait l'objet de nombreux travaux de recherches. Ces faisceaux donnent lieu à des phénomènes physiques différents suivant qu'ils soient continus ou à impulsions [1]. L'utilisation des faisceaux d'énergie continue nécessite un balayage en surface ; le temps de séjour du faisceau en un point donné est compris entre $10^{-4}$ et $10^{-2} \mathrm{~s}$. Ce temps est long comparé au temps de réponse thermique de la région superficielle du matériau (épaisseur $=10 \mu \mathrm{m}$ ) mais, il est comparable au temps de réponse thermique de toute l'épaisseur de l'échantillon. Dans ce cas, une partie de la chaleur s'évacue en profondeur pendant le temps d'irradiation. Le recuit est dit en régime de flux thermique [1]. Le recuit par faisceaux laser continu consiste donc à chauffer la couche endommagée à haute température afin d'avoir une croissance épitaxique en phase solide complète lors de l'irradiation. Le matériau n'étant jamais fondu lors du traitement, la rediffusion des impuretés est donc négligeable ce qui semblait tout à fait favorable pour la technologie des dispositifs électroniques à semiconducteurs. Pourtant, les composants électroniques réalisés avec ce procédé ont révélé des performances inférieures à celles réalisées par un procédé classique [2]. En conséquence, plusieurs travaux de caractérisation de matériaux implantés et recuits laser continu ont été effectués pour étudier l'influence des différents paramètres pouvant intervenir dans le recuit laser (température du substrat, puissance laser, recouvrement des lignes de balayage, etc...). Ces études [3] montrent l'introduction de défauts par le faisceau laser. La première évidence expérimentale en a été donnée par A. Chantre et al. [4] dans le silicium vierge.

Les résultats reportés dans la littérature [5] concernant le recuit par faisceaux laser continu de GaAs montrent que la recristallisation en phase solide des couches implantées est complètement obtenue dans le cas d'une zone faiblement endommagée. Si la couche implantée devient amorphe, le phénomène de recristallisation est plus difficile à obtenir surtout lorsque le substrat GaAs est à température ambiante ce qui entraîne l'apparition de forts gradients thermiques et des lignes de glissement. Cependant, l'utilisation d'un préchauffage du substrat $\left(500^{\circ} \mathrm{C}\right)$ et des 
vitesses de balayages faibles $(<1 \mathrm{~mm} / \mathrm{s})$ réduit les gradients thermiques et élimine les lignes de glissement. Cette dernière approche a donné une bonne mobilité des porteurs mesurée sur des matériaux GaAs implantés. Nissim et Gibbons [6], ont montré que les seuils de dommage peuvent être dépassés si la décomposition de la surface (évaporation d'arsenic) s'accomplit lors du recuit dans un environnement oxydant (oxygène ou air). Dans ces conditions, la puisance incidente de recuit peut être augmentée jusqu'à induction de températures de l'ordre de 1000 à $1200^{\circ} \mathrm{C}$ sur le substrat. La décomposition de la surface s'accompagne de la formation d'un oxyde $\left(\beta-\mathrm{Ga}_{2} \mathrm{O}_{3}\right)$ et agit comme une relaxation des contraintes imposées par la puissance incidente élevée. Mais jusqu'à présent aucune étude de défauts induits dans GaAs par recuit laser continu n'a été faite. Ceci fait l'objet de notre présent travail.

\section{Description des techniques expérimentales.}

2.1 Préparation des ÉChantillons. - Dans le but d'éliminer l'effet des défauts préexistant dans le matériau, on a choisi du GaAs LPE non implanté et de type $\mathrm{n}$. La concentration des porteurs libres mesurée par la méthode $C(V)$ est de $10^{17} \mathrm{~cm}^{-3}$. Les différentes opérations de réalisation des diodes, à partir du matériau de départ, sont les suivantes :

- Prise des contacts ohmiques.

- Attaque chimique de la surface (qq $\mu \mathrm{m}$ ).

- Evaporation d'or pour réaliser le contact Schottky.

Pour les échantillons traités, l'évaporation du métal se fait immédiatement après recuit laser.

2.2 LE MONTAGE DU RECUIT LASER. - La figure 1 montre le système de balayage laser argon utilisé au laboratoire d'application laser au centre de Spectra Physics de la région Rhône Alpes. La puissance incidente maximale que l'on peut atteindre est de $20 \mathrm{~W}$. Une lentille focalisante est placée entre le laser et deux miroirs perpendiculaires de réflexion, entraînés par des moteurs pas à pas, permettent le contrôle de la vitesse de balayage, ainsi que la distance de séparation latérale entre les lignes de

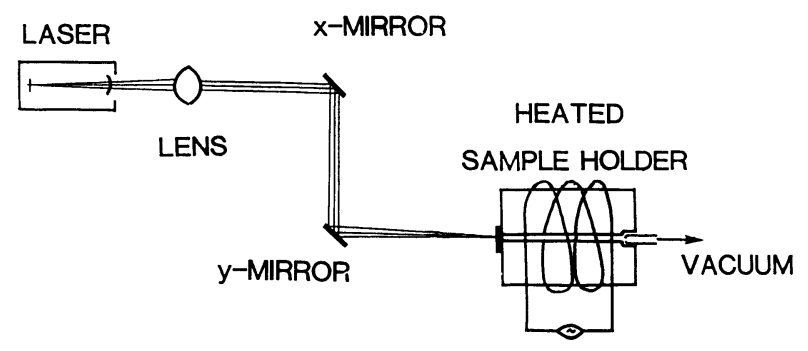

Fig. 1. - Schéma général du système Laser.

[CW scanning argon laser system.] balayage (recouvrement). Tous les paramètres du traitement sont contrôlés par ordinateur. Le matériau semiconducteur à traiter est placé sur une porteéchantillon chauffant et est plaqué sur celui-ci par dépression. La température maximale de ce porteéchantillon est de $450{ }^{\circ} \mathrm{C}$. Le traitement laser s'effectue à l'air libre avec toutefois la possibilité d'emploi d'une enceinte pour le recuit sous atmosphère de gaz inerte. Les paramètres importants qui interviennent lors d'un recuit par faisceaux laser continu sont :
$P i$ : Puissance incidente.
To : Température initiale du substrat.
$d$ : Recouvrement (distance entre deux lignes de balayage).
$v \quad:$ Vitesse de balayage.
$r \quad$ : Rayon du spot.

La puissance de fusion notée $P m$ dépend des paramètres $T o, d$ et $v$; pour cela, une étude systématique pour la détermination des seuils de fusion est effectuée sur des échantillons tests avant chaque traitement.

Compte tenu de cette remarque on a introduit le paramètre $P r$, Puissance relative : $P r=P i / P m$, qui exprime le rapport de la quantité d'énergie déposée par rapport à celle nécessaire pour atteindre la fusion. Les autres conditions expérimentales sont maintenues égales à : $T o=25^{\circ} \mathrm{C}, \quad v=10 \mathrm{~cm} / \mathrm{s}$, $r=25 \mu \mathrm{m}, d=20 \mu \mathrm{m}$. Ces paramètres donnent une puissance de fusion $P m=6,5 \mathrm{~W}$.

2.3 MÉThodes D’ANALYSE : DLTS/DLOS. L'étude électrique des défauts dans nos échantillons a été réalisée à l'aide d'un banc DLTS qui se compose essentiellement d'un capacimètre Boonton à entrées différentielles et d'une détection synchrone ORTHOLOC double phase à large bande passante. Les spectres DLTS sont enregistrés à des températures variant entre $77 \mathrm{~K}$ et $340 \mathrm{~K}$ avec un coefficient d'émission de $173,88 \mathrm{~s}^{-1}$, la durée du pulse de remplissage est fixée à $50 \mu \mathrm{s}$. Le déroulement automatique des mesures DLTS est assuré par un calculateur HP 9826. Le schéma complet du dispositif utilisé est donné en [7]. La méthode DLOS (Deep level optical spectroscopy) est utilisée pour déterminer les sections efficaces de photoionisation optique des pièges détectés. Les différents modes de fonctionnement de la DLOS utilisées sont exposés dans la publication [8].

\section{Résultats expérimentaux.}

Les caractéristiques électriques des différentes diodes après recuit laser ont été comparées à celles d'une diode témoin sur le matériau vierge. La hauteur de barrière $\phi_{\mathrm{bn}}$ du contact métal semiconducteur calculée par la méthode $I(V)$ ne varie pas avec la puissance $\operatorname{Pr}$ du faisceau laser et reste égale à $0,7 \mathrm{~V}$. Les courbes $C(V)$ donnent une valeur extra- 
polée de $V_{\mathrm{bi}}$ supérieure à $\phi_{\mathrm{bn}}$ et est égale à $1 \mathrm{~V}$. Cet écart signifie qu'il y a à la surface du matériau une couche interfaciale probablement d'oxyde, entraînant un léger décalage de la courbe $C^{-2}(V)$ donc une valeur extrapolée de $V_{\text {bi }}$ plus élevée. D'autre part la valeur de la concentration moyenne des porteurs $n(x)$ a faiblement diminuée par rapport à celle mesurée sur la diode non irradiée. Le tableau I rassemble ces résultats.

Tableau I. - Valeurs de la hauteur de barrière $\phi_{\mathrm{bn}}$, de la tension de diffusion $V_{\mathrm{bi}}$ et de la concentration moyenne des porteurs $n(x)$ calculées pour les différentes puissances Pr utilisées.

[Values of barrier height $\phi_{b n}$, potential diffusion $V_{\mathrm{bi}}$ and carrier concentration $n(x)$ computed for different power of the laser scan Pr.]

\begin{tabular}{cc|c|c|c|c}
$\begin{array}{c}\text { Puissance relative } \\
P r\end{array}$ & 0 & 0,46 & 0,54 & 0,61 & 0,69 \\
\hline $\begin{array}{c}\text { Hauteur de barrière } \\
\phi_{\text {bn }}(\mathrm{V})\end{array}$ & 0,66 & 0,76 & 0,61 & 0,73 & 0,67 \\
\hline $\begin{array}{c}\text { Tension de diffusion } 1 \\
V_{\text {bi }}(\mathrm{V})\end{array}$ & 1,03 & 1 & 1,05 & 1,01 \\
\hline $\begin{array}{c}\text { Zone désertée à } \\
W_{0}(\mu \mathrm{m})\end{array}$ & 0,124 & 0,154 & 0,140 & 0,136 & 0,148 \\
\hline
\end{tabular}

3.1 EXPÉRIENCES DLTS. - Sur la figure 2 sont représentés les spectres DLTS des pièges à électrons détectés après recuit laser continu pour différentes valeurs de la puissance $P r$ juste après traitement laser (de l'ordre de $24 \mathrm{~h}$ ). On observe plusieurs structures plus au moins larges qui évoluent au cours du temps pour donner naissance systématiquement à un défaut PL1 représenté sur la figure 3 pour différentes puissances $\operatorname{Pr}$ (il ne s'agit pas à proprement parler d'un défaut mais d'une famille donnant lieu à un continuum d'états, par simplification nous continuerons à parler du « défaut PL1 »). Le but de cette étude est de déterminer les propriétés de ce défaut «PL1». L'évolution temporelle de ces spectres dépend d'une façon complexe de la puissance $P r$ et sort du cadre de cette étude. Les pics détectés juste après irradiation dépendent de la puissance laser utilisée. Pour les faibles puissances $P r=0,46$, le spectre DLTS donne un fond continu sans toutefois faire apparaître de pics proprement dits. Pour $\operatorname{Pr}=0,54$, il y a apparition de deux pics très rapprochés, et à plus forte puissance le spectre donne un pic élargi autour de $T=200 \mathrm{~K}$. PL1 est observable dans tous les cas au bout d'un mois environ. Toutes nos expériences systématiques sont effectuées sur le pic PL1 stable. Les spectres de la figure 3 sont enregistrés avec un taux d'émission de

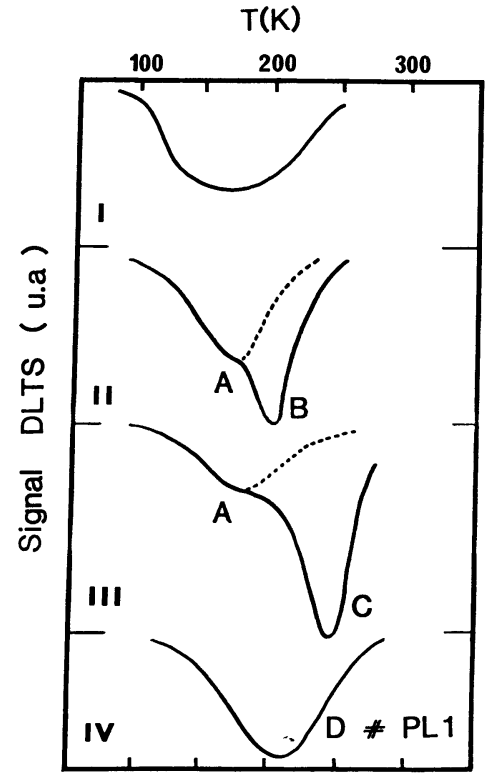

Fig. 2. - Spectres DLTS enregistrés après 15 jours de stockage des diodes montrant l'évolution du piège PL1 en fonction de la puissance laser $\operatorname{Pr}:$ I : 0,46 , II : 0,54 , III : 0,61 , IV : $0,69 . V_{\mathrm{r}}=-1 \mathrm{~V}, V_{\mathrm{p}}=+1 \mathrm{~V}, t_{\mathrm{p}}=1 \mathrm{~ms}$, $e=12,22 \mathrm{~s}^{-1}$.

[DLTS Spectrum of diodes stocked for 15 days showing the evolution of the defect PL1 with laser power $\operatorname{Pr}: \mathrm{I}$ : 0.46 , II : 0.54 , III : 0.61 , IV : $0.69 . \quad V_{\mathrm{r}}=-1 \mathrm{~V}$, $V_{\mathrm{p}}=+1 \mathrm{~V}, t_{\mathrm{p}}=1 \mathrm{~ms}, e=12.22 \mathrm{~s}^{-1}$.]

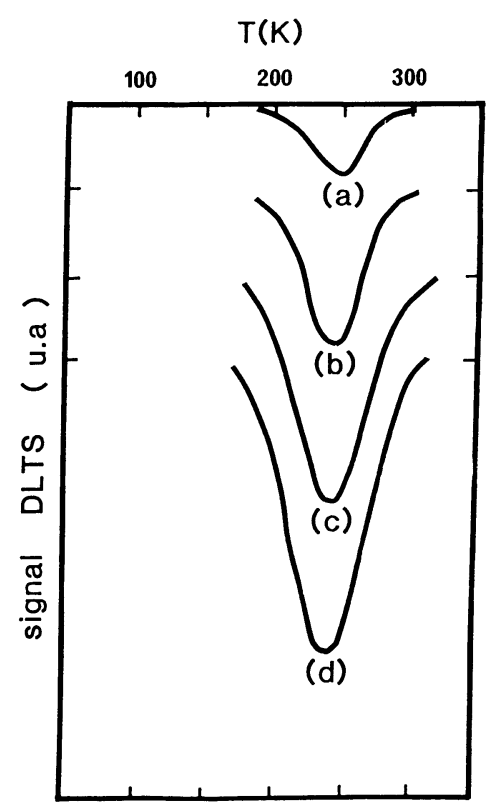

Fig. 3. - Spectres DLTS d'échantillons recuits Laser cw pour différentes puissances $\operatorname{Pr}:$ (a) 0,46, (b) 0,54, (c) 0,61, (d) 0,69 . $V_{\mathrm{r}}=-1 \mathrm{~V}, \quad V_{\mathrm{p}}=+2,4 \mathrm{~V}, \quad t_{\mathrm{p}}=50 \mu \mathrm{s}$, $e=173,88 \mathrm{~s}^{-1}$.

[DLTS spectrum recorded on samples annealed at different powers $P r$ : (a) 0.46 , (b) 0.54 , (c) 0.61 , (d) 0.69 . $V_{\mathrm{r}}=-1 \mathrm{~V}, V_{\mathrm{p}}=+2.4 \mathrm{~V}, t_{\mathrm{p}}=50 \mu \mathrm{s}, e=173.88 \mathrm{~s}^{-1}$.] 
$173,88 \mathrm{~s}^{-1}$, la polarisation inverse étant fixée à $-1 \mathrm{~V}$ et la valeur du pulse de tension directe est de $+2,4 \mathrm{~V}$ pour une durée de $50 \mu \mathrm{s}$. Le signal DLTS correspondant au défaut PL1 augmente avec la puissance laser. La largeur du pic PL1 prouve d'emblée qu'il ne s'agit pas d'un défaut classique ayant une énergie bien déterminée dans le gap mais plus généralement d'une famille de défauts. Ceci sera confirmé par la suite : on ne peut pas appliquer l'analyse classique, une "signature " calculée dans ce cas n'ayant aucune signification. La figure 4 donne les spectres DLTS de l'échantillon irradié à $P r=0,54$, la tension de polarisation inverse étant maintenue égale à $0 \mathrm{~V}$.

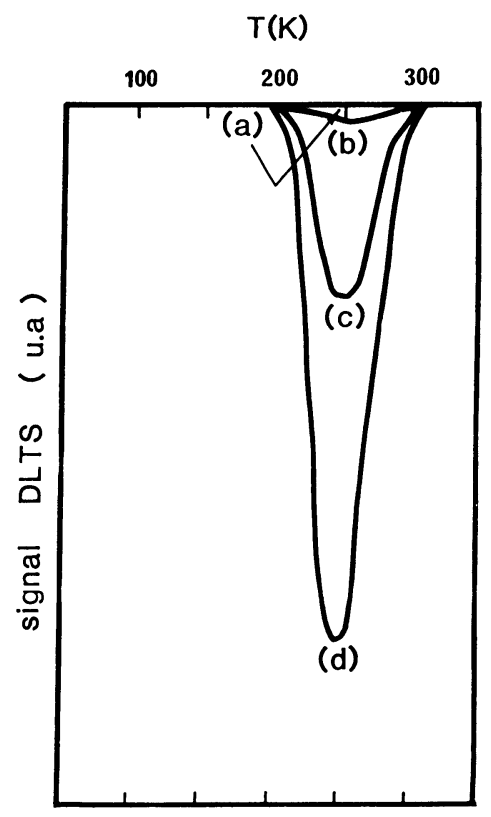

Fig. 4. - Spećtres DLTS de l'échantillon irradié à $P r=0,54 ; P m=6,5 \mathrm{~W}$ (voir texte) fonction de la tension de pulse directe $V_{\mathrm{p}}$. (a) $0,6 \mathrm{~V}$, (b) $0,65 \mathrm{~V}$, (c) $0,75 \mathrm{~V}$, (d) $1 \mathrm{~V} . e=173,88 \mathrm{~s}^{-1}, V_{\mathrm{r}}=0 \mathrm{~V}, t_{\mathrm{p}}=50 \mu \mathrm{s}$.

[DLTS spectrum of the sample annealed at $\operatorname{Pr}=0.54$; $P m=6.5 \mathrm{~W}$ (see text) as a function of the pulse excitation $V_{\mathrm{p}} \cdot V_{\mathrm{p}}=$ (a) $0.6 \mathrm{~V}$, (b) $0.65 \mathrm{~V}$, (c) $0.75 \mathrm{~V}$, (d) $1 \mathrm{~V}$. $e=173.88 \mathrm{~s}^{-1}, V_{\mathrm{r}}=0 \mathrm{~V}, t_{\mathrm{p}}=50 \mu \mathrm{s}$.]

En faisant varier l'amplitude du pulse de remplissage $V_{\mathrm{p}}$, on remarque que le signal DLTS n'apparaît qu'à partir de $V_{\mathrm{p}}=+0,65 \mathrm{~V}$, et continue à augmenter jusqu'à $V_{\mathrm{p}}=+2,4 \mathrm{~V}$ où il sature.

Ceci montre que le centre PL1 est localisé à la surface : cette augmentation du signal DLTS pour des tensions directes croissantes est typique de défauts d'interface dans des MIS. En fonction de la tension inverse (Fig. 5(a)), on observe deux phénomènes : d'une part une diminution rapide de l'amplitude des spectres avec $V_{\mathrm{r}}$ aisément explicable par le fait qu'il s'agit d'une famille de défauts de surface

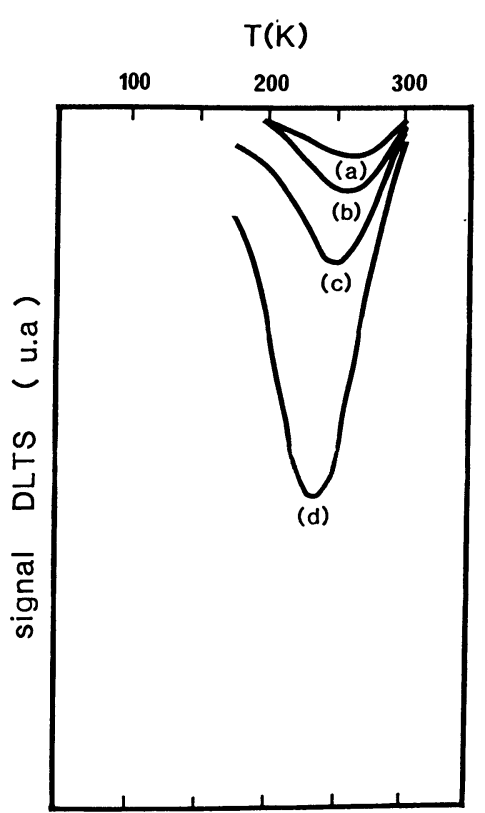

Fig. 5. - (a): Spectres DLTS de l'échantillon irradié à $P r=0,54$, fonction de la tension inverse $V_{\mathrm{r}}:$ (a) $-3 \mathrm{~V}$, (b) $-2 \mathrm{~V}, \quad$ (c) $-1 \mathrm{~V}, \quad$ (d) $0 \mathrm{~V}$. $e=173,88 \mathrm{~s}^{-1}$, $V_{\mathrm{p}}=+2,4 \mathrm{~V}, t_{\mathrm{p}}=50 \mu \mathrm{s}$.

[(a) DLTS spectrum of the sample annealed at $\mathrm{Pr}=0.54$ as a function of the reverse bias $V_{\mathrm{r}}(\mathrm{a})-3 \mathrm{~V}$, (b) $-2 \mathrm{~V}$, (c) $-1 \mathrm{~V}, \quad$ (d) $0 \mathrm{~V} . \quad e=173.88 \mathrm{~s}^{-1}, \quad V_{\mathrm{p}}=+2.4 \mathrm{~V}$, $t_{\mathrm{p}}=50 \mu \mathrm{s}$.]

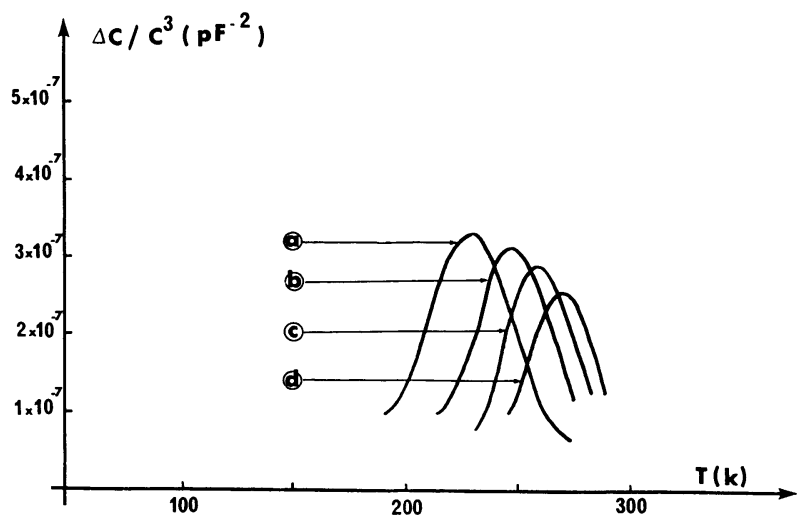

Fig. 5. - (b) : Spectres normalisés à $\Delta C / C^{3}$.

[(b) normalized spectrum at $\Delta C / C^{3}$.]

(voir discussion 4), d'autre part un décalage avec $V_{\mathrm{r}}$ vers les hautes températures. Ce décalage est « rigide », c'est-à-dire s'effectue sans déformation notable des spectres normalisés, comme on peut le voir de façon plus claire sur la figure $5 \mathrm{~b}$ des mêmes spectres normalisés. Tout se passe donc comme si l'énergie d'activation (ou la position énergétique) du piège augmentait avec le champ électrique. Nous avons effectué des mesures de DLOS sur ce défaut. Il est à noter que ce type de mesure sur des 


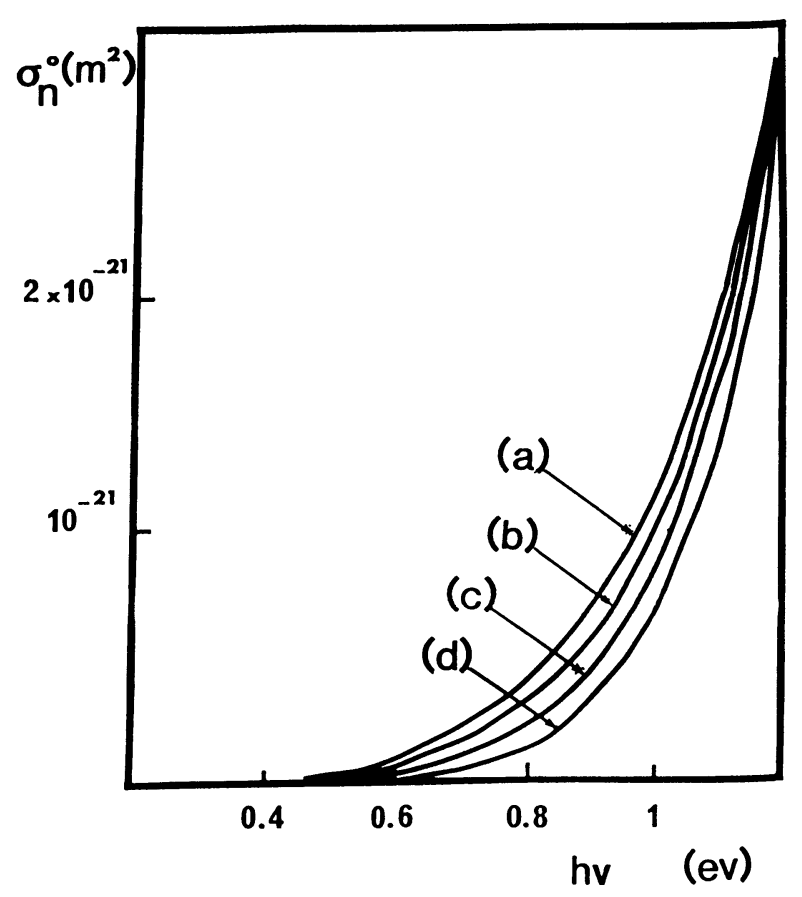

Fig: 6. - Sections de photoionisation optique $\sigma_{\mathrm{n}}(h \nu)$ mesurées par DLOS électrique du piège PL1 pour différentes tensions inverses $V_{\mathrm{r}}$ : (a) $0 \mathrm{~V}$, (b) $-1 \mathrm{~V}$, (c) $-2 \mathrm{~V}$, (d) $-3 \mathrm{~V}, \mathrm{~T}=78 \mathrm{~K}, V_{\mathrm{p}}=+2,4 \mathrm{~V}, t_{\mathrm{p}}=1 \mathrm{~ms}$.

[Photoionization cross section curves $\sigma_{\mathrm{n}}(h \nu)$ measured by DLOS for the PL1 electron trap at different reverse bias $V_{\mathrm{r}}$ : (a) $0 \mathrm{~V}$, (b) $-1 \mathrm{~V}$, (c) $-2 \mathrm{~V}$, (d) $-3 \mathrm{~V}$. $T=$ $78 \mathrm{~K}, V_{\mathrm{p}}=+2,4 \mathrm{~V}, t_{\mathrm{p}}=1 \mathrm{~ms}$.]

continuums est tout à fait légitime comme il a été démontré par Kamenicki sur l'interface $\mathrm{Si} / \mathrm{SiO}_{2}$ [9]. On remarque donc un décalage du spectre de photoionisation vers les hautes énergies quand la tension inverse appliquée augmente (voir Fig. 6).

\subsection{ANALYSE DES TRANSITOIRES DE CAPACITÉ. -} Les cinétiques de transitoires de capacité sont enregistrées à différentes températures, dans tout le domaine de température du pic PL1 sur une période de $1,2 \mathrm{~s}$, avec un échantillonnage de $6,5 \mathrm{~ms}$. Les cinétiques enregistrées montrent que la variation $C(t)$ de la capacité est logarithmique dans un large domaine de température $(80 \mathrm{~K}-200 \mathrm{~K})$, figure 7 . Audelà de $200 \mathrm{~K}$, les cinétiques ne sont plus logarithmiques (nous considérons les cinétiques non logarithmiques lorsque le coefficient de corrélation par analyse des moindres carrés est inférieur à 0,95).

Dans ce cas le dépouillement classique des pics DLTS (signature, concentration du piège) n'est évidemment plus valable. Il faut donc recourir à d'autres types d'analyse fondés sur un modèle de continuum d'états. L'étude complète de ces cinétiques est exposée dans le paragraphe 4.

3.3 ANALYSE SIMS ET ELLIPSOMÉTRIE. - L'état de la surface a changé à cause du traitement. Nous

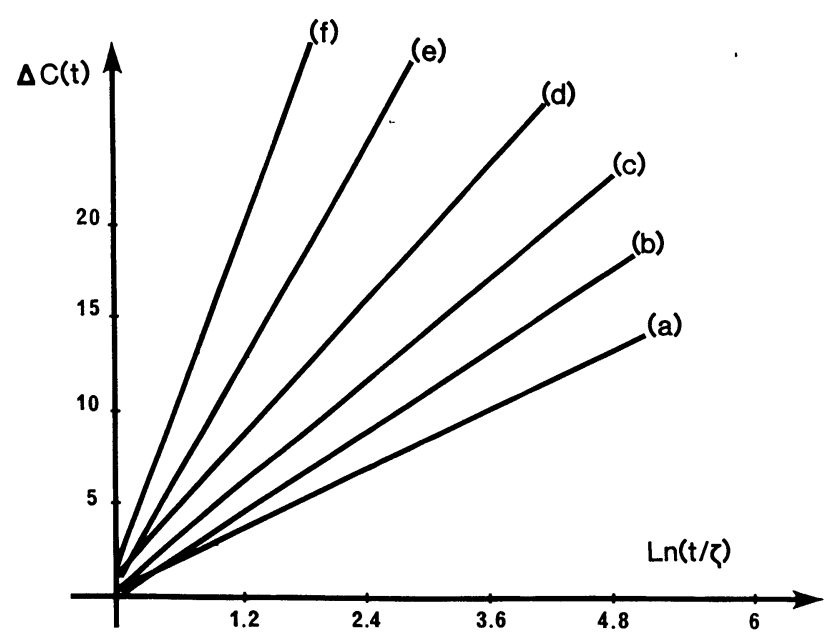

Fig. 7. - Variation de la capacité en fonction de $1 \mathrm{n}(t / \tau)$ correspondante au piège PL1 pour différentes températures : $T=$ (a) $100 \mathrm{~K}$, (b) $120 \mathrm{~K}$, (c) $130 \mathrm{~K}$, (d) $150 \mathrm{~K}$, (e) $170 \mathrm{~K}$, (f) $190 \mathrm{~K}, V_{\mathrm{r}}=0 \mathrm{~V}, V_{\mathrm{p}}=+2,4 \mathrm{~V}$, $t_{\mathrm{p}}=1 \mathrm{~ms}$.

[Capacitance transient for PL1 trap plotted as a function of $1 \mathrm{n}(t / \tau)$ recorded at different temperatures. $T=(\mathrm{a})$ $100 \mathrm{~K}$, (b) $120 \mathrm{~K}$, (c) $130 \mathrm{~K}$, (d) $150 \mathrm{~K}$, (e) $170 \mathrm{~K}$, (f) $190 \mathrm{~K}, V_{\mathrm{r}}=0 \mathrm{~V}, V_{\mathrm{p}}=+2.4 \mathrm{~V}, t_{\mathrm{p}}=1 \mathrm{~ms}$.]

avons étudié celle-ci avant et après recuit laser par ellipsométrie. Cette méthode permet de connaître l'épaisseur d'une couche d'oxyde moyennant certains modèles. La mesure est effectuée avec un laser $\mathrm{He}$ $\mathrm{Ne}$ à une longueur d'onde $\lambda=632,8 \mathrm{~nm}$. Nous considérons ici que sur le matériau GaAs d'indice $n_{\mathrm{s}}=3,856$, existe une couche d'oxyde uniforme et de composition homogène d'incide $n_{0}=1,96$, correspondant à l'indice d'une couche d'oxyde de gallium $\left(\mathrm{Ga}_{2} \mathrm{O}_{3}\right)$; (modèle de monocouche) [10]. On a analysé la couche d'oxyde déjà existante sur un matériau témoin non recuit avant et après traitement chimique. Le temps qui s'écoule entre le traitement et l'observation est de l'ordre de $5 \mathrm{~min}$. Les principaux résultats de cette étude préliminaire sont donnés sur le tableau II. Il en découle qu'une couche d'oxyde natif de $25 \AA$ se forme automatiquement dès que la surface du matériau est en contact avec l'oxygène de l'air. Ceci est en accord avec les résultats de C. W. Wilmsen [11]. Deux échantillons recuits ont été étudiés correspondant respectivement à $\operatorname{Pr} 1=0,46$ et $\operatorname{Pr} 2=0,54$. L'épaisseur de la couche d'oxyde formée juste après irradiation laser pour la puissance $\operatorname{Pr} 1$ n'a pas changé. Pour la puissance $\operatorname{Pr} 2$, l'effet de l'irradiation laser apparaît : on a mesuré une couche de $60 \AA$. Il est important de noter que ces résultats sont à prendre avec beaucoup de précaution. En effet, le modèle utilisé peut ne pas être adéquat à partir du moment où la couche d'oxyde formée n'est plus homogène et uniforme sur toute la surface. Les études SIMS effectuées sur les échantillons irradiés démontrent la présence de cette 
Tableau II. - Epaisseur de la couche d'oxyde mesurée sur la surface du matériau GaAs par ellipsométrie après différents traitements chimiques pour l'échantillon non recuit et recuit aux puissances Pr1 et Pr2.

[Thickness of oxyde layer measured at the surface of GaAs material by ellipsometry after the different chemical treatment for unannealed and annealed samples at $\operatorname{Pr} 1$ and $\operatorname{Pr} 2$.]

Traitement chimique effectué avant la mesure et avant irradiation
Mesure sur échantillon non irradié
Mesure après irradiation à

$$
\operatorname{Pr} 1=0,46 \quad \operatorname{Pr} 2=0,54
$$

\begin{tabular}{lcccc}
\hline Sans & $90 \AA$ & - & - \\
\hline Attaque $\mathrm{HCl}(37 \%)$ & $40 \AA$ & - & - \\
\hline
\end{tabular}

Attaque

$\left(\mathrm{H}_{2} \mathrm{SO}_{4}+\mathrm{H}_{2} \mathrm{O}_{2}+\mathrm{H}_{2} \mathrm{O}\right)$

$25 \AA$

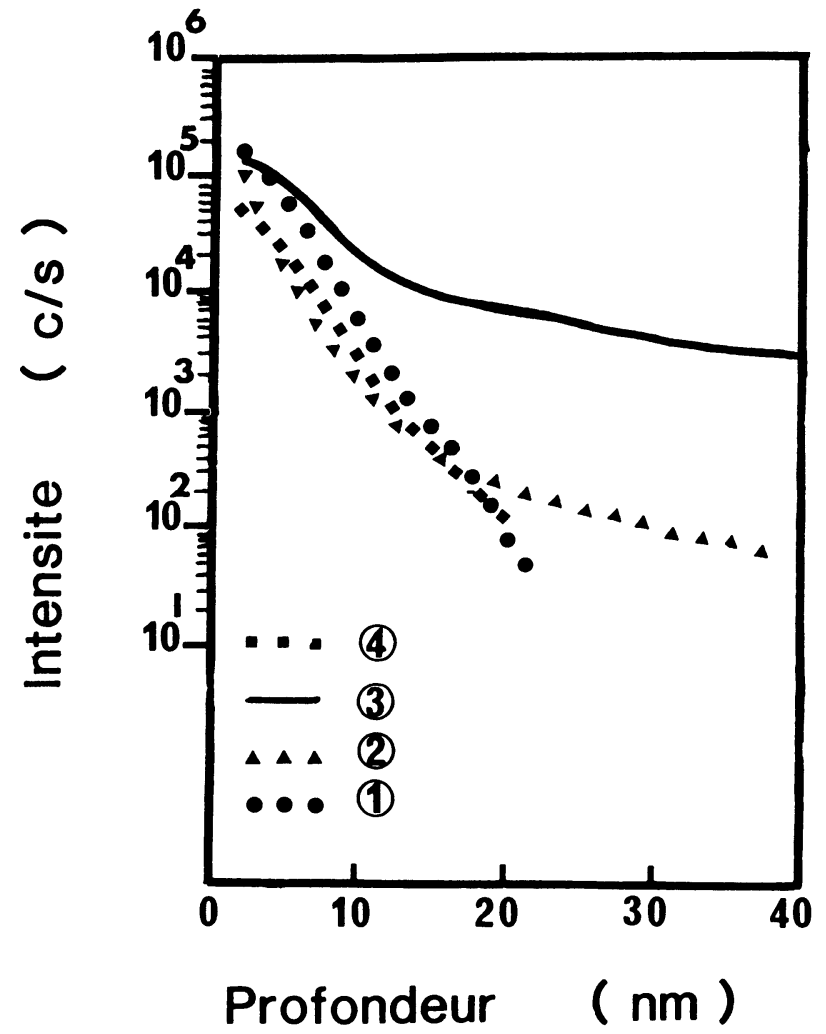

Fig. 8. - Spectre d'analyse SIMS (recherche de l'oxygène) des échantillons irradiés à différentes puissances $\operatorname{Pr}$ : (1) 0,46, (2) 0,54, (3) 0,61, (4) 0,69 .

[SIMS Spectrum (oxygen signal) of the samples irradiated with various laser powers $\operatorname{Pr}:$ (1) 0.46, (2) 0.54, (3) 0.69, (4) 0.69 .]

couche d'oxyde (voir Fig. 8), la réponse de l'oxygène au voisinage de la surface est importante et diminue de 3 ordres de grandeurs entre 0 et $40 \mathrm{~nm}$ de la surface pour les deux échantillons étudiés. Afin de corréler la présence du défaut «PL1 » et de la couche d'oxyde, nous avons effectué des mesures DLTS sur des échantillons traités laser et ensuite décapé par $\mathrm{HCl}$. Aucun signal DLTS n'est alors enregistré.

3.4 ETUDE DU VIEILLISSEMENT DES STRUCTURES SCHOTTKYS. - Les résultats obtenus à présent suggèrent que le défaut PL1 provienne de la formation d'une couche d'oxyde qui s'est formée lors du recuit et transformée sous la couche d'or. Pour vérifier si cet oxyde est différent de celui d'une surface libre du matériau, une partie d'un échantillon irradié a été laissée en contact avec l'air ambiant pendant une durée de 30 jours, sur l'autre partie des diodes Schottky ont été formées juste après irradiation. Le résultat montre que pour cette puissance laser $(\operatorname{Pr}=0,69)$, le même défaut apparaît sur les deux parties. Donc, l'oxyde formé sous la couche d'or est identique à celui qui s'est formé sur la surface libre donnant naissance au défaut PL1. Nous avons pu vérifier que PL1 était présent en concentration très faible lorsque les Schottky sont faites sur des surfaces préparées et laissées suffisamment longtemps à l'air avant vaporisation. D'autre part le défaut PL1 a même été finalement détecté dans des diodes anciennes effectuées suivant des procédures normales de préparation. Sur des échantillons non irradiés laissés à l'air nous avons vérifié que ce défaut n'existait pas initialement. Compte tenu des faibles épaisseurs d'or utilisées (pp $100 \AA$ A), il semble donc que l'oxygène ait pu diffuser à travers les défauts de la couche d'or et finalement former une couche d'oxyde donnant naissance au défaut PL1.

En conséquence on peut dire que ce centre est dû de façon parfaitement générale à la formation d'une couche d'oxyde sur GaAs, cette oxydation a lieu à température ambiante et est activée par le traitement laser.

\section{Discussion.}

La présence d'une cinétique logarithmique dans les transitoires de capacité est révélateur de la présence 
d'un continuum d'états dans la bande interdite de GaAs [12]. En effet, si l'on suppose une distribution énergétique $N_{T}(E)$ d'états localisés, le transitoire de capacité à la température $T$ entre deux temps $t_{1}$ et $t_{2}$ est donné par :

$$
\begin{aligned}
& C\left(t_{2}\right)-C\left(t_{1}\right)=A \cdot \int_{E_{\mathrm{v}}}^{E_{\mathrm{c}}} N_{T}(E) \times \\
& \quad \times\left[\exp \left(-e_{\mathrm{n}}(E) \cdot t_{2}\right)-\exp \left(-e_{\mathrm{n}}(E) \cdot t_{1}\right)\right] \cdot \mathrm{d} E
\end{aligned}
$$

avec

$$
e_{\mathrm{n}}=e_{\mathrm{n} 0} \cdot \exp (-E / k T)
$$

où $E_{\mathrm{v}}$ et $E_{\mathrm{c}}$ sont les énergies de la bande de valence et de conduction et $e_{\mathrm{n} 0}$ est le taux d'émision du piège [13].

Le coefficient de proportionalité $A$ dépend de la répartition spatiale des pièges à l'intérieur du semiconducteur. Si $N_{T}(E)$ varie peu sur un intervalle d'énergie de $3 k T$, il est aisé de montrer que l'équation (1) s'écrit :

$$
\Delta C(t)=A \cdot k T \cdot N_{T}\left(E_{\mathrm{c}}-E_{\mathrm{w}}\right) \cdot \ln \left(t_{2} / t_{1}\right)
$$

où

$$
E_{\mathrm{w}}=k T \cdot \ln \left[\ln \left(t_{2} / t_{1}\right) / e_{\mathrm{n} 0}\left(t_{2}-t_{1}\right)\right]
$$

Il est alors commode d'imaginer que le transitoire de capacité à la température $T$ est dû à la réponse des pièges situés dans la fenêtre énergétique large de

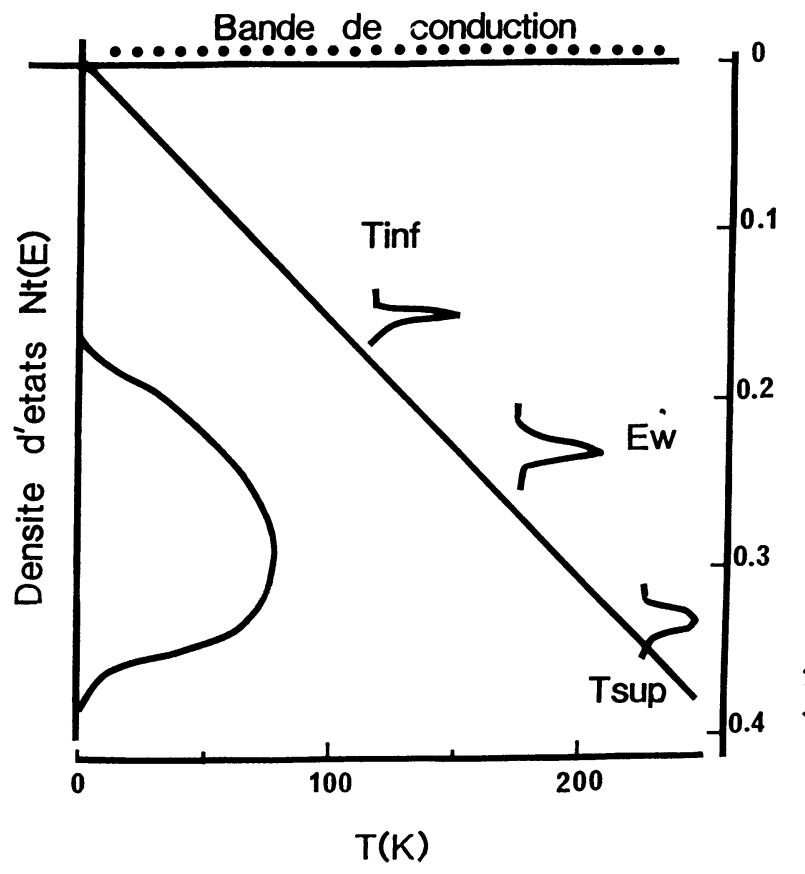

Fig. 9. - Représentation symbolique du déplacement de la fenêtre de constante de temps $E_{\mathrm{w}}$ en fonction de la température.

[Symbolic representation for the displacement of $E_{\mathrm{w}}$ versus temperature.] quelques $k T$ à une distance $E_{\mathrm{w}}(T)$ du bas de la bande de conduction de GaAs. Cette fenêtre balaye le gap supérieur de GaAs lorsque la température varie (voir Fig. 9) [12]. Dans les dépouillements de transitoires que nous effectuons, c'est en fait $\Delta C(t)=C\left(n t_{0}=t\right)-C\left(t_{0}\right)$ qui est enregistré pour différentes $T$ soit, d'après (3) :

$$
\delta \Delta C(t)=A \cdot k T \cdot N_{T} \cdot \ln (t / \tau)
$$

où $\tau=t_{0}$ si le modèle est valide. La figure 7 montre que les résultats expérimentaux sont en très bon accord avec ce modèle théorique. Lorsque la fenêtre énergétique $E_{\mathrm{w}}$ commence à sortir du continuum (pour les températures $T_{\text {inf }}$ et $T_{\text {sup }}$ de la figure 9), les cinétiques perdent leur allure logarithmique pour devenir de plus en plus proche d'une exponentielle, ce qui est confirmé par l'expérience $\left(T_{\text {inf }}<80 \mathrm{~K}\right.$ et $\left.T_{\text {sup }}>200 \mathrm{~K}\right)$. Si les pièges sont localisés à la surface du semiconducteur, la constante $A$ de l'équation (1) est donnée par :

$$
A=C^{3} / \varepsilon_{\mathrm{s}} \cdot C_{0 \mathrm{X}} \cdot N_{\mathrm{D}}
$$

où $C$ est la capacité d'équilibre, $C_{0 \mathrm{x}}$ la capacité d'une couche isolante entre GaAs et le métal, $N_{\text {D }}$ le dopage du semiconducteur et $\varepsilon_{\mathrm{s}}$ sa constante diélectrique [12]. Le tableau III montre que les valeurs du rapport $\Delta C / C^{3}$ au maximum des spectres DLTS pour différentes tensions inverses appliquées. Cette valeur étant sensiblement constante, ce continuum d'états se trouve donc localisé près de la surface de GaAs. Des équations (5) et (6), nous tirons une densité d'états d'interfaces $N_{T}=1,25 \times$ $10^{11} \mathrm{eV}^{-1} \cdot \mathrm{cm}^{-2}$. L'influence du champ électrique sur les spectres de DLTS pose un problème plus fondamental. L'activation de l'émission par champ électrique (Frenkel-Poole, effet tunnel...) conduit à un déplacement de la fenêtre de constante de temps $E_{\mathrm{w}}$ plus profondément dans la bande interdite. Par

Tableau III. - Valeurs de $\Delta c / c^{3}$ calculées au maximum du pic PL1 pour différentes valeurs de la tension inverse $V_{\mathrm{r}}$.

[Values of $\Delta c / c^{3}$ computed at the maximum of the $P L 1$ peack for different values of the reverse bias $\left.V_{\mathrm{r} .}\right]$

\begin{tabular}{cc}
$\begin{array}{c}\text { Tension inverse } \\
V_{\mathrm{r}}(\mathrm{V})\end{array}$ & $\Delta C / C^{3}\left(\mathrm{pF}^{-2}\right)$ \\
\hline 0 & $3,33 \times 10^{-7}$ \\
\hline-1 & $3,11 \times 10^{-7}$ \\
\hline-2 & $2,94 \times 10^{-7}$ \\
\hline-3 & $2,56 \times 10^{-7}$
\end{tabular}


exemple, en cas d'abaissement de barrière $\Delta E$ (effet Frenkel-Poole), $E_{\mathrm{w}}$ est déplacée d'une quantité constante $\Delta E$ [12]. Les résultats de la figure $5 \mathrm{~b}$ sont en contradiction évidente avec cette prédiction. Une analyse plus fastidieuse de l'effet tunnel de défauts localisés dans une couche fine isolante entre $\mathrm{GaAs}$ et le métal ainsi que de l'effet de CUT-OFF [14] dû à l'intersection du niveau de Fermi de GaAs et de la fenêtre de constante de temps conduit à la même contradiction: ceci fera l'objet d'une publication ultérieure. Nous sommes donc conduits à penser que cette bande d'impuretés localisée à l'interface occupe un niveau énergétique qui dépend du champ électrịque de surface $F_{\mathrm{m}}$ : plus ce champ $F_{\mathrm{m}}$ est élevé, plus le niveau est profond dans le gap de GaAs. Ce résultat est d'ailleurs en accord avec le déplacement des spectres DLOS avec la tension inverse appliquée (Fig. 6). Le fait que ce type de défauts apparaisse lors d'évaporation d'ors de faibles épaisseurs est un renseignement très précieux car il nous ramène à des résultats déjà observés dans le silicium. Lorsque des couches très fines d'or sont évaporées sur des plaquettes de $\mathrm{Si}$, les cinétiques d'oxydation de $\mathrm{Si}$ sont alors accélérées sur plusieurs ordres de grandeurs : des oxydes $\mathrm{SiO}_{2}$ peuvent même être obtenus à température ambiante [15]. On peut supposer que l'or joue le même rôle de catalyseur d'oxydation de GaAs. Une couche de $\mathrm{Ga}_{2} \mathrm{O}_{3}$ croîtrait donc en quelques jours, avec un défaut d'interface $\mathrm{GaAs} / \mathrm{Ga}_{2} \mathrm{O}_{3}$ dont l'émission est inhibée par le champ électrique. Un modèle microscopique de la nature électrique de tels pièges est à l'étude.

\section{Remerciements.}

Nous remercions Monsieur A. Gagnaire qui a bien voulu faire l'analyse d'ellipsométrie au laboratoire d'électronique, automatique et mesures électriques de l'Ecole Centrale de Lyon, et Madame Dubois pour les analyses SIMS à la sonde ionique CAMECA IMS $3 \mathrm{~F}$ du laboratoire. Les expériences au laboratoire d'application laser « Spectra-Physics » CNET ont été réalisées avec la collaboration de Monsieur P. Pierrard.

\section{Bibliographie}

[1] SEdgwick, T., J. Electrochem. Soc. : Solid-State Sci. Technol. 130 (1983) 484.

[2] Sheng, N. H., Mizuta, M., Merz, J. L., Laser and Electron Beam Solid Interactions and Materials Processing, 1981 Eds. J. F. Gibbons, L. D. Hess and T. W. Sigmon (North Holland, NW) 1981, p. $155-162$.

[3] Johnson, N. M., Gold, R. B., Gibbons, J. F.; Phys. Lett. 34 (1979) 704.

[4] Chantre, A., Kechouane, M., Bois, D., 12th Int. Conf. Defects in Semiconductors C.A.J. Ammerlaan Ed. (North Holland, Amsterdam) 1982 p. 547-552.

[5] Cullis, A. G., Rep. Prog. Phys. 48 (1985) 1233, printed in Great Britain.

[6] Nissim, Y. and Gibbons, J. F., Laser and Electron Beam Solid Interactions and Materials Processing, J. F. Gibbons, L. D. Hess and T. W. Sigmon Ed. (Amsterdam North-Holland) (1980) pp. 275-80.
[7] Pelloie, J. L., Guillot, G., Nouailhat, A. and Antolini, A. G., J. Appl. Phys. 59 (1986) 1536.

[8] Bremond, G., Guillot, G., Nouailhat, A. and Picoli, G., J. Appl. Phys. 59 (1986) 2038.

[9] KaMIEnIECKI, E., Solid State Electron. 16 (1973) 1487.

see also Pierret, R. F. and Roesner, B. B., Appl. Phys. Lett. 24 (1974) 366.

[10] WEAST, R. C., CRC Handbook of Chemistry and Physics (CRC Press, Cleveland, Ohio) 1975.

[11] Wilmsen, C. W., Physics and Chemistry of III-V Compound Semiconductor Interface.

[12] Rosencher, E., CoppaRd, R. and BoIs, D., J. Appl. Phys. 57 (1985) 2823.

[13] Lang, D., J. Appl. Phys. 45 (1974) 3023.

[14] Rosencher, E. and Coppard, R., J. Appl. Phys. 55 (1984) 971.

[15] Cros, A., Derrien, J. and Salvan, F., Surf. Sci. 110 (1981) 471. 